Communication

\title{
Development of a Novel Pipette Tip-Aided Cell Cloning Method for The Effective Isolation of Genome-Edited Porcine Cell
}

\author{
Masahiro Sato ${ }^{1,}{ }^{*}$, Issei Saitoh ${ }^{2}$, Eri Akasaka ${ }^{1}$, Emi Inada $^{3}$
}

1. Section of Gene Expression Regulation, Frontier Science Research Center, Kagoshima University, Kagoshima 890-8544, Japan; E-Mails: masasato@m.kufm.kagoshima-u.ac.jp; stylistics777@yahoo.co.jp

2. Division of Pediatric Dentistry, Department of Oral Health Sciences, Course for Oral Life Science, Graduate School of Medical and Dental Sciences, Niigata University, 951-8514 Niigata, Japan; EMail: isaito@dent.niigata-u.ac.jp

3. Department of Pediatric Dentistry, Graduate School of Medical and Dental Sciences, Kagoshima University, 890-8544 Kagoshima, Japan; E-Mail: inada@dent.kagoshima-u.ac.jp

* Correspondence: Masahiro Sato; E-Mail: masasato@m.kufm.kagoshima-u.ac.jp

Academic Editor: Joep Geraedts

Special Issue: Genome Editing

OBM Genetics

2021, volume 5 , issue 1

doi:10.21926/obm.genet.2101126
Received: October 11, 2020

Accepted: March 04, 2021

Published: March 10, 2021

\begin{abstract}
Direct colony cloning of adherent mammalian cells using rings or dilution cloning has been used frequently for obtaining stable transfectants after gene delivery. As an alternative to these methods, successful isolation of the cells in a single colony is possible by placing a trypsin-immersed small paper disk onto the colony and subsequently picking up the paper with the assumption that it carries the trypsinized cells. However, the cloning success using this technique largely relies on the cell type used. In the present study, a novel, simple, and non-invasive technique for the isolation of cells from single colonies using a disposable pipette tip was developed. Using this technique, success was achieved in isolating the clonal populations of genome-edited porcine fibroblastic cells with $100 \%$ efficiency after cotransfection with the clustered, regularly interspaced, short palindromic repeats-CRISPR
\end{abstract}

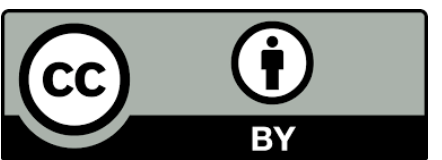

(C) 2021 by the author. This is an open access article distributed under the conditions of the Creative Commons by Attribution License, which permits unrestricted use, distribution, and reproduction in any medium or format, provided the original work is correctly cited. 
associated protein 9 (CRISPR/Cas9)-based genome editing components [for targeting the porcine GGTA1 that encodes $\alpha$-1,3-galactosyltransferase ( $\alpha$-GaIT)] and the piggyBac-based gene delivery components [to enable efficient chromosomal integration of the transgene carrying the cDNA of enhanced green fluorescent protein (EGFP)]. A toxin-based, drug-free

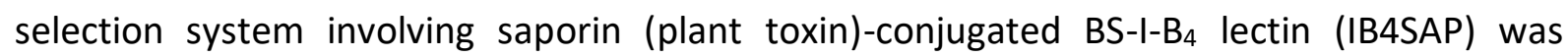
employed in the present study. Since IB4SAP binds specifically to the cell-surface $\alpha$-Gal epitope (synthesized by $\alpha$-GaIT), it is supposed that treatment with IB4SAP theoretically eliminates the untransfected or genome-edited porcine cells with a mono-allelic knockout (KO) phenotype, while all the surviving clones have a bi-allelic GGTA1 mutation. A total of 16 clones were isolated in the present study, all of which exhibited loss of the $\alpha$-Gal epitope (a cell-surface carbohydrate synthesized by $\alpha$-GalT), suggesting that all the clones had a biallelic KO phenotype. Moreover, 75\% of these clones expressed EGFP uniformly, while the remainder had mosaic or no EGFP expression. These findings indicate the fidelity of the developed pipette tip-aided cell cloning approach for the efficient isolation of genomeedited porcine fibroblast clones.

\section{Keywords}

$\alpha$-Gal epitope; $\alpha$-1,3-galactosyltransferase; cell cloning; colony; disposable pipette tip; trypsin; genetically modified cells; genome editing; CRISPR/Cas9; toxin selection

\section{Introduction}

Isolation of clonal cells from a single colony is an essential step in the process of obtaining pure populations of stably-transfected clones after gene transfer and the subsequent drug selection. Direct colony cloning of adherent mammalian cells using rings and dilution cloning is performed frequently in the field of genetic engineering [1-2]. However, these methods have certain drawbacks, such as the inability to establish clonal expansion when the cells are seeded at a low density in dilution cloning. It is often difficult to handle steel rings as they require sterile grease to ensure adhesion between the rings and secure the dish substratum. These rings require washing and autoclave-mediated sterilization after each usage, which is time-consuming and laborious. Another technique to isolate individual small colonies is the use of small paper disks [3]. This method involves the isolation of cells using trypsin-dipped disks (made of Whatman ${ }^{\circledR} 3 \mathrm{MM}$ paper) and offers the advantage of isolating several clones simultaneously. Furthermore, it enables the simultaneous collection of several cells $(>100)$ comprising a colony and seeding them onto the surface of 48-well plates in a relatively crowded environment, which is beneficial for cell growth, probably due to the autocrine mechanisms involving growth accelerating factors. However, even with the paper disk method, poor growth of certain porcine cells isolated after cloning has been observed. It was inferred that these porcine cells might have been highly sensitive to contact with the $3 \mathrm{MM}$ paper used in the disks.

Advances in the genome-editing technology, such as the development of clustered, regularly interspaced short palindromic repeats-CRISPR associated protein 9 (CRISPR/Cas9), have enabled the rapid and convenient production of gene-modified (GM) cells and animals by disrupting a 
target gene following the insertion or deletion of nucleotides (indels) [4-9]. In the present study, the aim was to disrupt the porcine GGTA1 locus encoding $\alpha$-1,3-galactosyltransferase ( $\alpha$-GalT) using the CRISPR/Cas9 technology. $\alpha$-GalT is a key enzyme involved in the synthesis of a cellsurface carbohydrate, referred to as the $\alpha$-Gal epitope, which is responsible for the hyper-acute rejection of the grafted tissues in human-to-pig xenotransplantation [10-11]. Therefore, porcine fibroblasts lacking functional $\alpha$-GalT are useful as nuclear donors for the somatic cell nuclear transfer-based production of $\alpha$-GalT-negative pigs. In addition, engineering the GGTA1 locus may be beneficial for developing a novel negative selection-based method for isolating the GM porcine cell clones containing a gene of interest (GOI) within their genome, as $\alpha$-GalT deficiency is not essential for the survival and function of porcine cells $[12,13]$. In order to verify this hypothesis, porcine fibroblasts were co-transfected with two components, i.e., the CRISPR/Cas 9 components and the piggyBac (PB)-based gene delivery components, in the present study. PB is a transposon system that enables efficient chromosomal integration of a GOI [e.g., a transgene carrying the sequence for enhanced green fluorescent protein (EGFP)] in various types of mammalian cells, including porcine cells [14-20]. Subsequently, the transfected cells were selected through a short incubation (30 min to $2 \mathrm{~h}$ ) in a solution containing BS-I-B lectin (IB4) conjugated with the plant toxin saporin (hereafter referred to as IB4SAP). IB4 is known to bind specifically to the $\alpha-G a l$ epitope expressed on the cell surface of almost all mammalian cells (except for human and Old World monkey cells) [21, 22]. The incubation treatment, therefore, enables the elimination of unedited porcine cells and the cells with mono-allelic knockout (KO) phenotypes as these cells continue to express the $\alpha-G$ al epitope on their surfaces [12-14]. When the IB4SAP-treated cells were seeded on a dish containing a normal medium, the surviving colonies were observed to be $\alpha$ Gal epitope-negative and frequently carried the GOI within their genome [13, 20, 23].

In the present study, isolation of the clonal populations of GM porcine fibroblasts [lacking the $\alpha$-Gal epitope expression and expressing the GOI (EGFP) continuously] was attempted using a novel single-colony isolation technique involving the collection of trypsinized cells from a colony using a disposable $200-\mu \mathrm{L}$ pipette tip.

\section{Materials and Methods}

The experiments described ahead were performed in agreement with the guidelines of the Kagoshima University Committee on Recombinant DNA Security (No. 25035; dated 30 May 2018).

\subsection{CRISPR/Cas9-Related Agents and Plasmid Vectors}

In order to prepare ribonucleoproteins (RNPs), first, a guide RNA (gRNA) capable of recognizing a 20-bp sequence (5'-GAG AAA ATA ATG AAT GTC AA-3') spanning the translation initiation codon (ATG) upstream of the protospacer adjacent motif (PAM) sequence ( $A G G$ ) on the 4th exon of the porcine GGTA1 (encoding $\alpha$-GalT) was designed (Figure 1A). A single gRNA (sgRNA) synthesized by Integrated DNA Technologies, Inc. (IDT; Coralville, lowa, USA) was used as the Alt-R ${ }^{\mathrm{TM}}$ CRISPR crRNA product. The crRNA and tracrRNA (purchased from IDT) were combined for annealing to generate the sgRNA, followed by the addition of recombinant Cas9 protein (TaKaRa Bio, Inc., Ohtsu, Shiga, Japan) to produce the RNPs, according to the method reported by Ohtsuka et al. [24]. The prepared RNPs contained the Cas9 protein $(50 \mathrm{ng} / \mu \mathrm{L})$ and the sgRNA ( $200 \mathrm{ng} / \mu \mathrm{L})$. 
A
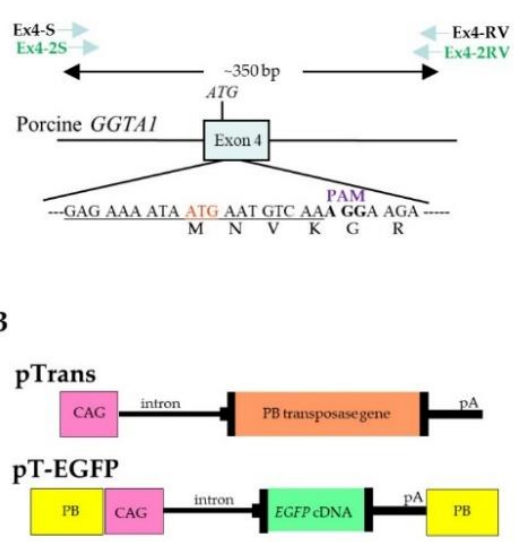

C

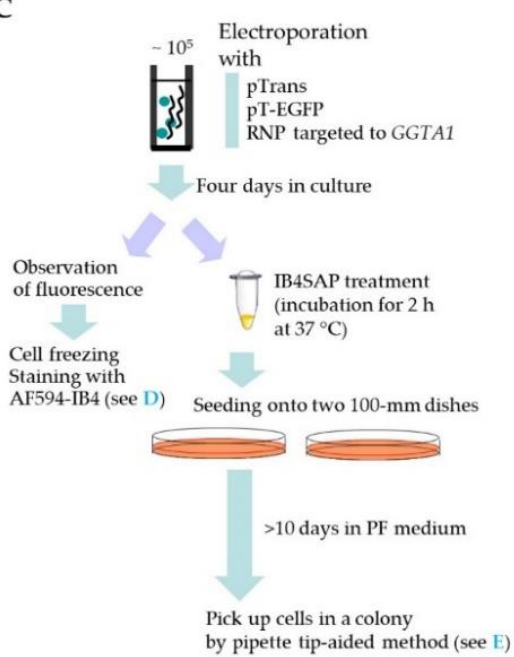

D

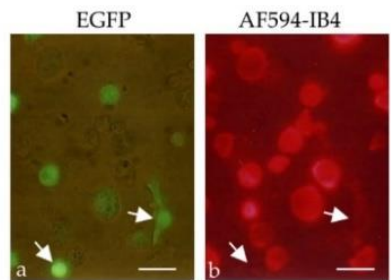

E

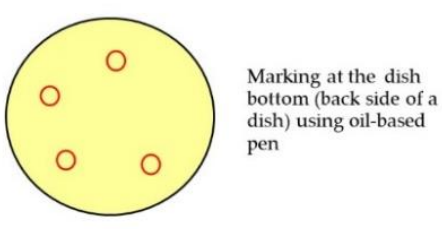

Remove medium and wash with DPBS. Then, add
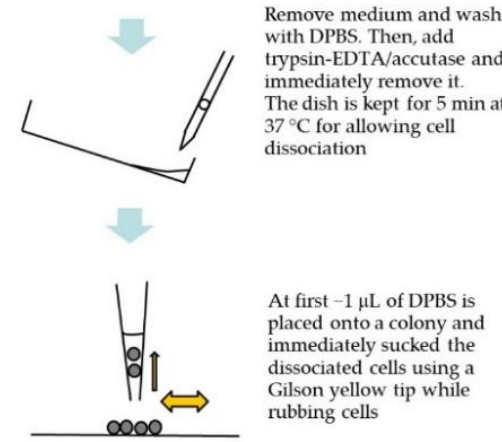

At first $-1 \mu \mathrm{L}$ of DPBS is placed onto a colony an immediately sucked the dissociated cells using Gilson yellow tip while rubbing cells

Figure $1 \mathrm{~A}$. Structure of exon 4 of porcine GGTA1. The sequence recognized by the gRNA is underlined. ATG indicates the translation initiation site, and the amino acid sequence is depicted below the nucleotide sequence. The primer sets [Ex4-S/Ex4-RV for the first polymerase chain reaction (PCR) and Ex4-2S/Ex4-2RV for the nested PCR] are provided above the sequence. AGG (depicted in bold) indicates the PAM site. B. Schematic representation of the piggyBac-based transposon vectors used in the present study. The plasmid backbone is not depicted in the figure. CAG, cytomegalovirus enhancer + chicken $\beta$-actin promoter; $p A$, poly $(A)$ sites; EGFP cDNA, enhanced green fluorescent protein CDNA; $\mathrm{PB}$, acceptor site in the piggyBac system. $\mathbf{C}$. Flowchart of the experiments for testing the feasibility of the novel cell colony isolation system using a 200- $\mu \mathrm{L}$ pipette tip. Four days after transfection with the ribonucleoprotein (RNP; targeted to GGTA1), pTrans, and pT-EGFP, the cells were treated with $\mathrm{BS}-\mathrm{I}-\mathrm{B}_{4}$ lectin conjugated with saporin (IB4SAP) for a short duration and then cultured in the PF medium for $>10$ days. The resultant colonies were propagated for molecular biology and cytochemical analyses. D. Staining of the transfected cells (4 days after transfection with RNP and piggyBac-related vectors) with Alexa Fluor 594 (AF594)-labeled BS-I-B 4 lectin (IB4). A few cells expressed EGFP (arrows in a) and concomitantly exhibited a loss of the $\alpha$-Gal epitope [caused by the mutations in GGTA1] (arrows in b). Fluorescence was observed under UV and visible light. Scale bar $=20 \mu \mathrm{m}$. E. Flowchart of the procedure for pipette tip-aided cell cloning. 
The plasmid vectors pTrans and pT-EGFP [23] used in the present study are illustrated in Figure 1B. Briefly, pTrans facilitates the expression of $P B$ transposase via the chicken $\beta$-actin gene-based promoter CAG [25]. pT-EGFP is derived from a pPB-based vector containing two PB acceptors with inverted repeats, and it carries an EGFP cDNA expression unit (CAG promoter + EGFP CDNA + poly(A) sites). All plasmids were grown in Escherichia coli $\mathrm{DH} 5 \alpha$ and subsequently purified using a MACHEREY-NAGEL plasmid purification kit (\#740615.10; TaKaRa Bio, Inc.), as described previously by Sato et al. [26].

\subsection{Cell Culture and Transfection}

Testis-derived porcine fibroblasts [27] were cultured in Dulbecco's modified Eagle's medium or Ham's F-12 medium (\#048-29785; Wako Pure Chemical Industries, Ltd., Osaka, Japan) supplemented with $10 \%$ fetal bovine serum (FBS) and $1 \%$ antibiotic-antimycotic solution (\#A5955; Sigma-Aldrich Co. Ltd., St. Louis, MO, USA) [hereafter referred to as the PF medium] at $37{ }^{\circ} \mathrm{C}$ in $5 \%$ $\mathrm{CO}_{2}$ atmosphere.

As schematically depicted in Figure $1 \mathrm{C}$, the porcine fibroblasts $\left(\sim 3 \times 10^{5}\right)$ were co-transfected in $100 \mu \mathrm{L}$ of the Ingenio ${ }^{\circledR}$ Electroporation Solution (\#MIR50111; TaKaRa Bio, Inc.) containing pTrans $(2 \mu \mathrm{g})$, pT-EGFP $(2 \mu \mathrm{g})$, and RNPs $(2 \mu \mathrm{L})$ through nucleofector-based electroporation (Lonza GmbH, Köln, Germany) under the electric field strength defined as V023 (experimental group). The control group comprised the cells $\left(\sim 3 \times 10^{5}\right)$ that were co-transfected in $100 \mu \mathrm{L}$ of a solution containing pTrans $(2 \mu \mathrm{g})$ and pT-EGFP $(2 \mu \mathrm{g})$ under the same electric conditions that were used for the experimental group. After the gene transfer, the porcine fibroblasts were seeded onto a 60mm gelatin-coated tissue-culture dish (\#4010-020; Iwaki Glass Co. Ltd., Tokyo, Japan) containing the PF medium and cultured as described previously for four days.

After four days of culture, the cells were examined for EGFP fluorescence to evaluate the transfection efficiency, just prior to the IB4SAP treatment. Fluorescent images of a randomly selected area (with $>400$ cells) were captured and used for calculating the transfection efficiency (\%) by examining over 400 cells.

\subsection{IB4SAP Treatment}

As schematically depicted in Figure 1C, four days after transfection, the cells were trypsinized, and aliquots from the cell suspension were examined for EGFP-derived fluorescence using a fluorescence microscope (as described below). Subsequently, the cells were subjected to either freezing in CELLBANKER ${ }^{\circledR}$ 1, a serum-containing cryopreservation medium (\#CB011; TaKaRa Bio, Inc.), for future use, or staining with AF (Alexa Fluor) 594-labeled IB4 (hereafter referred to as AF594-IB4; 0.5 mg/mL; \#I21413; Invitrogen Co., Carlsbad, CA, US), as described below in further detail. Trypsinized cells $\left(\sim 10^{5}\right)$ were subjected to IB4SAP treatment by $2 \mathrm{~h}$ of incubation at $37^{\circ} \mathrm{C}$ in a $25 \mu \mathrm{L}$ solution containing 0.5-1.0 $\mu \mathrm{g}$ IB4SAP (\#IT-10; Advanced Targeting Systems, Inc., San Diego, CA, USA) in Dulbecco's modified phosphate-buffered saline without $\mathrm{Ca}^{2+}$ and $\mathrm{Mg}^{2+}$ (DPBS) and with $5 \% \mathrm{FBS}$ and $1 \mathrm{mM} \mathrm{CaCl} 2$ (hereafter referred as $\mathrm{DPBS} / \mathrm{FBS} / \mathrm{CaCl}_{2}$ ), using our previously reported method $[12,13]$. After treatment, the cells were placed directly in two 100-mm gelatin-coated dishes (\#4020-020; Iwaki Glass Co. Ltd.) containing $6 \mathrm{~mL}$ of PF medium and then cultured for an additional 10-14 days for clonal expansion. 


\subsection{Pipette tip-aided Cell Cloning}

As schematically presented in Figure 1E, the emerging colonies were marked at the bottom of the dish, washed with $4 \mathrm{~mL}$ DPBS, and then rinsed with $4 \mathrm{~mL}$ of a solution containing $0.25 \%$ trypsin-EDTA (\#25200056; Thermo Fisher Scientific, Inc., Waltham, MA, USA) and accutase (\#AT104; Innovative Cell Technologies, Inc., San Diego, CA, USA) in a ratio of 1:1 (v/v). After removing the trypsin-EDTA-accutase-containing solution, the colonies were allowed to dissociate in a brief incubation of $5 \mathrm{~min}$ at $37{ }^{\circ} \mathrm{C}$. The cells within each colony (marked using an oil-based pen) were aspirated using a $200-\mu \mathrm{L}$ pipette tip, and the isolated cells were placed in the wells of gelatincoated 48-well plates (\#380-048; Iwaki Glass Co. Ltd.) containing PF medium ( $200 \mu \mathrm{L}$ in each well). After cell collection, the 100-mm dish was filled with PF medium to resume cell growth. The cells seeded in the 48-well plates were subjected to step-wise propagation, in which certain cells were subjected to staining with AF594-IB4, while the remaining cells were either frozen or subjected to genomic DNA isolation for the detection of mutated alleles at the target loci.

\subsection{Polymerase Chain Reaction (PCR) and Sequencing for Detection of GGTA1 Mutations}

Genomic DNA was extracted by adding $30 \mu \mathrm{L}$ of lysis buffer [containing $0.125 \mu \mathrm{g} / \mathrm{mL}$ proteinase $\mathrm{K}, 0.125 \mu \mathrm{g} / \mathrm{mL}$ Pronase $\mathrm{E}, 0.32 \mathrm{M}$ sucrose, $10 \mathrm{mM}$ Tris- $\mathrm{HCl}(\mathrm{pH} 7.5), 5 \mathrm{mM} \mathrm{MgCl}$, and $1 \%[\mathrm{v} / \mathrm{v}]$ Triton $\mathrm{X}-100]$ to the cell pellets $\left(10^{3}\right.$ to $10^{4}$ cells), followed by vigorous shaking overnight at $37^{\circ} \mathrm{C}$ and subsequent extraction with phenol/chloroform. The supernatant was subjected to ethanolprecipitation, and the precipitated DNA was dissolved in $20 \mu \mathrm{L}$ of sterile water and stored at $4{ }^{\circ} \mathrm{C}$.

The resulting DNA samples $(1 \mu \mathrm{L})$ were first subjected to PCR amplification in a volume of $20 \mu \mathrm{L}$ using the primer set Ex4-S (5'-GCAAATTAAGGTAGAACGCA-3') and Ex4-RV (5'GCTGCCCCTGAGCCACAACG-3') for targeting the porcine GGTA1 and the PCR conditions described in a previous report [23]. In addition, parental unedited porcine fibroblasts were used as controls in the PCR analysis. After the first round of PCR, nested PCR was performed in a volume of $20 \mu \mathrm{L}$ using $2 \mu \mathrm{L}$ of the first round PCR products, the nested primer set Ex4-2S (5'CTCCTTAGCGCTCGTTGGCT-3') and Ex4-2RV (5'-GCAACTCTCTGGAATGCTTT- $3^{\prime}$ ), and the same PCR conditions used in the first PCR. The expected size of the resultant product was $\sim 350 \mathrm{bp}$. One microliter of this product was examined in $2 \%$ agarose gel, while the remaining product was subjected to purification using a NucleoSpin ${ }^{\circledR}$ Gel and a PCR Clean-up kit (\#U0609A; TaKaRa Bio, Inc.). Direct sequencing of the PCR products was performed using a customized DNA sequencing service (Eurofins Genomics K.K., Tokyo, Japan).

\subsection{Staining with AF594-IB4 and Detection of Fluorescence}

Staining of the cells (in the form of the cell suspension or cell sheet) with AF594-IB4 was performed by incubating the cells in DPBS/FBS/CaCl 2 containing $2 \mu \mathrm{g} / \mathrm{mL}$ of AF594-IB4 for $30 \mathrm{~min}$ at $4{ }^{\circ} \mathrm{C}$. After washing with DPBS containing $0.3 \% \mathrm{FBS}$, the cells were examined under a fluorescence microscope (BX60; Olympus, Tokyo, Japan) using the DM505 (BP460-490 and BA510IF; Olympus) and DM600 filters (BP545-580 and BA6101F; Olympus) for detecting EGFPderived green fluorescence and AF594-derived red fluorescence, respectively. Micrographs were obtained using a digital camera (FUJIX HC-300/OL; Fuji Film, Tokyo, Japan) attached to the 
fluorescence microscope, and the images were printed using a Mitsubishi digital color printer (CP700DSA; Mitsubishi, Tokyo, Japan).

\section{Results and Discussion}

The objective of the present study was to confirm the usefulness of the proposed novel pipette tip-aided method in the isolation of highly pure genome-edited cells. As schematically depicted in Figure $1 C$, porcine fibroblasts were first co-transfected with the CRISPR/Cas9 genome-editing components (RNPs targeted to GGTA1) and PB vectors (pTrans and PT-EGFP). Four days later, among the cells stained with AF594-IB4, 60\% of cells exhibited EGFP-derived fluorescence (arrows in Figure 1D-a), indicating successful transfection. Among these EGFP-expressing cells, a few cells presented the loss of the $\alpha$-Gal epitope expression, as evidenced by their inability to react with AF594-IB4 (arrows in Figure 1D-b). These cells were then selected using IB4SAP, which enabled the elimination of the $\alpha$-Gal epitope-expressing cells, including intact (unedited) cells and the cells with mono-allelic KO phenotypes (as depicted in Figure $1 \mathrm{C}$ ). Only the cells exhibiting a complete loss of the $\alpha$-Gal epitope (possibly caused by mutations in both the alleles of GGTA1, and is, therefore, representative of the cells with bi-allelic KO phenotype) survive after the IB4SAP treatment. After 10-14 days of the toxin-based selection, more than 20 surviving colonies (comprising $>300$ cells in each colony) per dish were visually discernible. On the contrary, no viable colonies were visible in the control dish, in which the cells were transfected with pTrans and pTEGFP prior to the IB4SAP treatment. In order to isolate the cells within a colony using a disposable 200- $\mu \mathrm{L}$ tip, each colony was marked at the bottom of a dish using an oil-based pen (upper panel of Figure 1E) and then, after the removal of the PF medium, the dish was washed with DPBS and finally rinsed with the trypsin-accutase mixture (middle panel of Figure $1 \mathrm{E}$ ). Subsequently, the dish was placed in an incubator at $37^{\circ} \mathrm{C}$ for $5 \mathrm{~min}$ to allow cell dissociation, after which the cells were confirmed to be completely dissociated ( $a$ vs. $b$ in Figure $2 A$ ). The dissociated cells were collected through aspiration of approximately $1 \mu \mathrm{L}$ of DPBS into the pipette tip and then placing the tip onto a colony. Then, the cells were sucked into the pipette tip while rubbing them with the tip (Figure $1 \mathrm{E}$, middle panel). These aspirated cells were then transferred to PF medium ( $200 \mu \mathrm{L}$ ) placed in a well of a 48-well plate (Figure 1E, lower panel). When the colony (from which the cells were isolated) was examined under an inverted microscope, it was observed that almost all cells had disappeared (Figure 2A-c, the area enclosed by dotted yellow lines), although a few cells were observed around the marginal area of the marked circle (arrows in Figure 2A-c). Figure 2A-d depicts the cells that were successfully transferred to the well of a 48-well plate after cell isolation. 
A
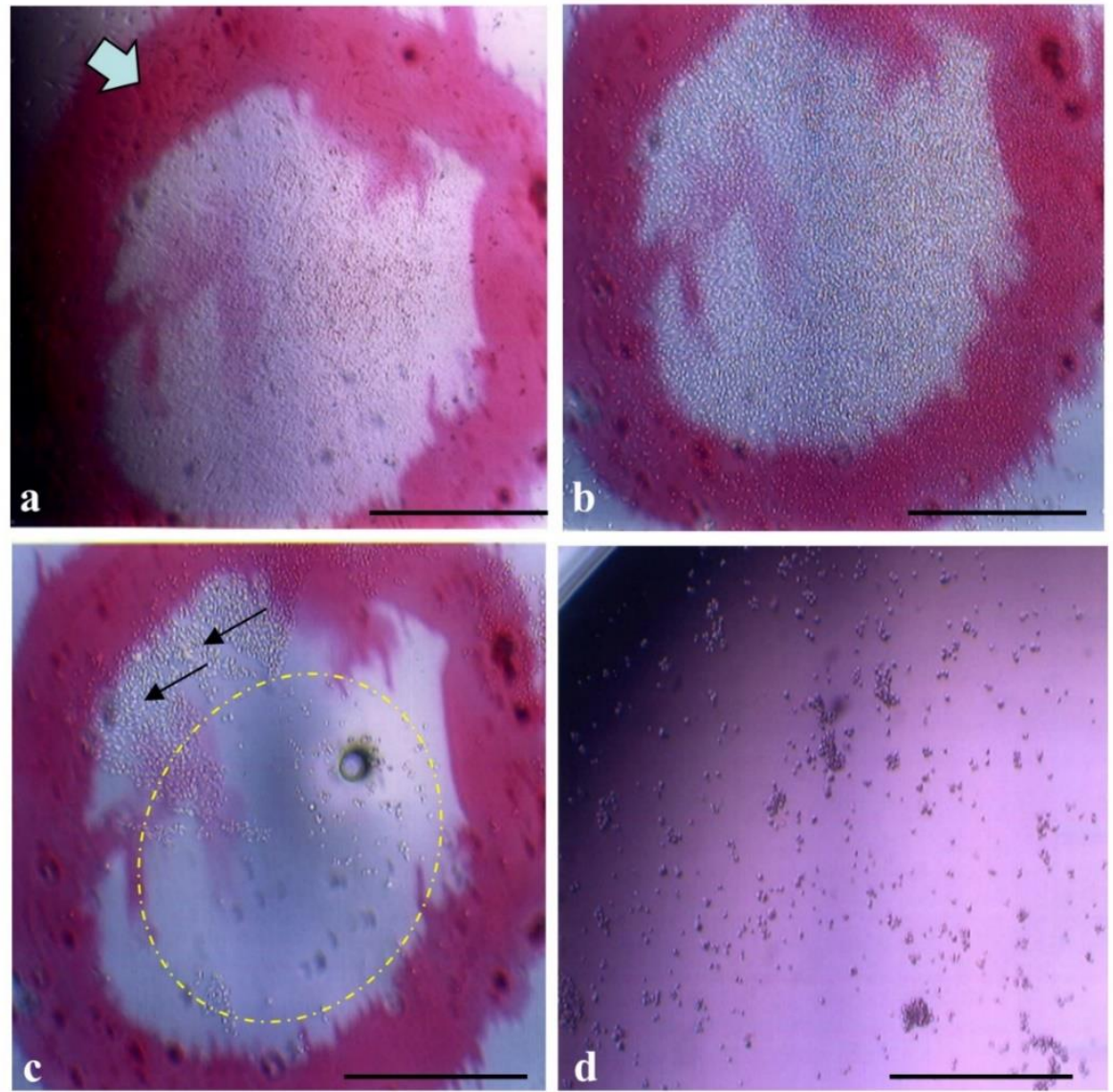

B

Phase

EGFP
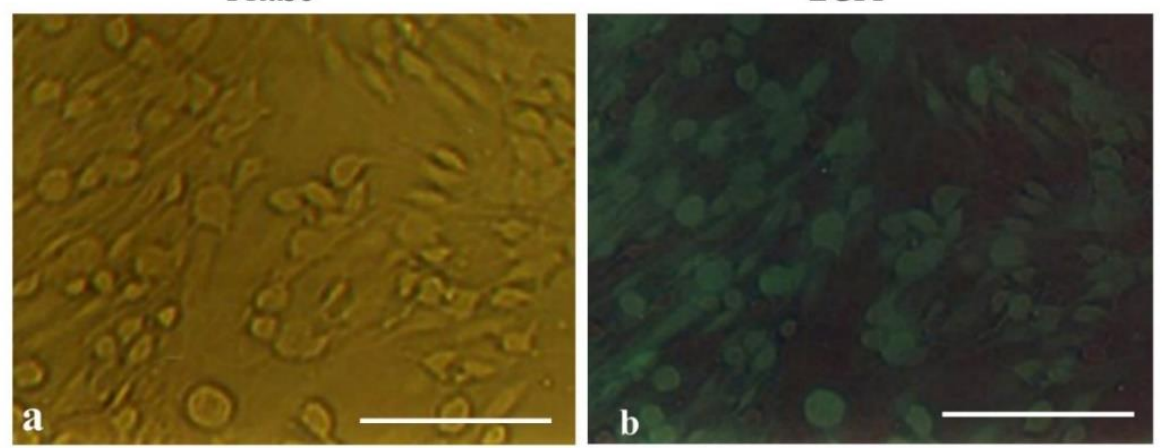

Figure 2 Porcine cell clones isolated from single colonies using pipette tip-aided cell cloning. A. A single colony prior to $(a, b)$ and after $(c, d)$ cell isolation. Prior to cell isolation, a single colony was marked in red (arrow in a) using an oil-based pen. After treatment with trypsin-EDTA-accutase mixture, cells demonstrated dissociation, evidenced by the appearance of rounded cells (b). When the dissociated cells were aspirated using a $200-\mu \mathrm{L}$ pipette tip, almost all cells were removed (depicted by the dotted yellow circle in c), with a few cells remaining at the marginal area (arrows in c). Isolated cells from single colonies were safely transferred to the wells in the 48-well plates. Single cells and a few aggregates comprising single cells were observed in the plate wells (d). Scale bar $=200 \mu \mathrm{m}$. B. Clone $\# 1$ collected using pipette tip-aided cell cloning of the colonies generated in the experiment illustrated in Figure 1C. Note that all the cells express EGFP-derived fluorescence (b). Scale bar $=50 \mu \mathrm{m}$. 
Finally, cell clones from 16 colonies were collected and successfully propagated. In Figure 2B, the images of one clone (Clone \#1) are depicted as a typical example. This clone expressed EGFP uniformly (Figure 2B-b). When all the isolated clones were screened for EGFP-derived fluorescence, almost all clones (75\%; 12/16) expressed EGFP uniformly, similar to Clone \#1 (Figure 3A-a; Table 1). Two clones (numbered 8 and 12) exhibited mosaic expression of EGFP, i.e., they exhibited a mixture of EGFP-positive and EGFP-negative cells (as exemplified by Clone \#8 in Figure 3A-c; Table 1). Two other clones (numbered 9 and 13) were negative for EGFP-derived fluorescence (Table 1). Staining with AF594-IB4 demonstrated that all clones were negative for the expression of the $\alpha$ Gal epitope (Figure 3A-b,d; Table 1). In contrast, control cells transfected with pTrans and pT-EGFP exhibited a mosaic EGFP expression while exhibiting ubiquitous $\alpha$-Gal epitope expression (Figure $3 A-e, f)$. These findings indicated that the proposed IB4SAP-based selection approach is quite useful in isolating the $\alpha$-Gal-negative porcine cells.

A

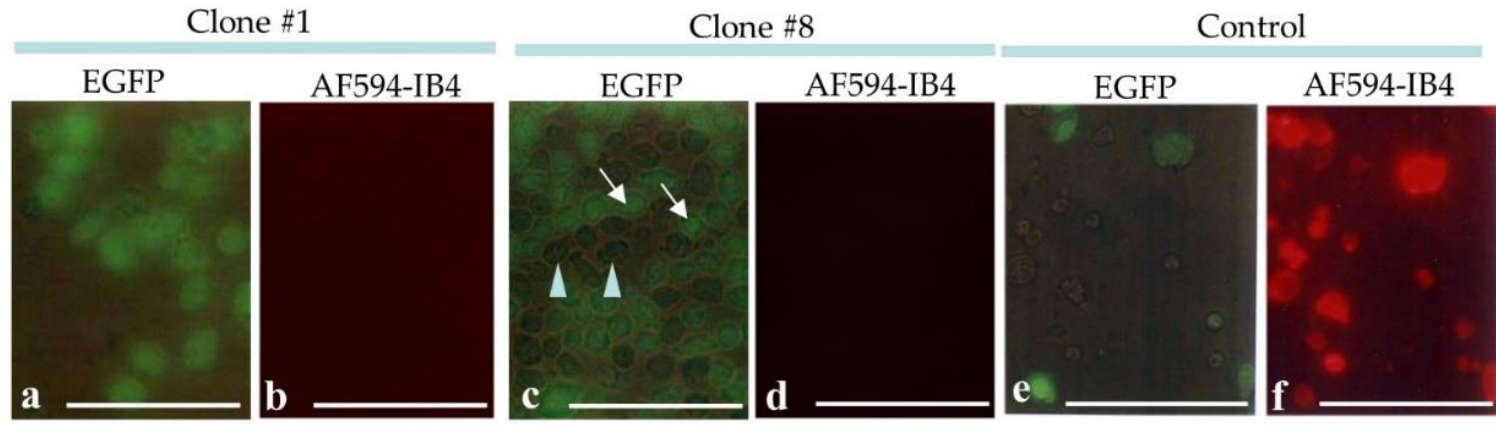

B
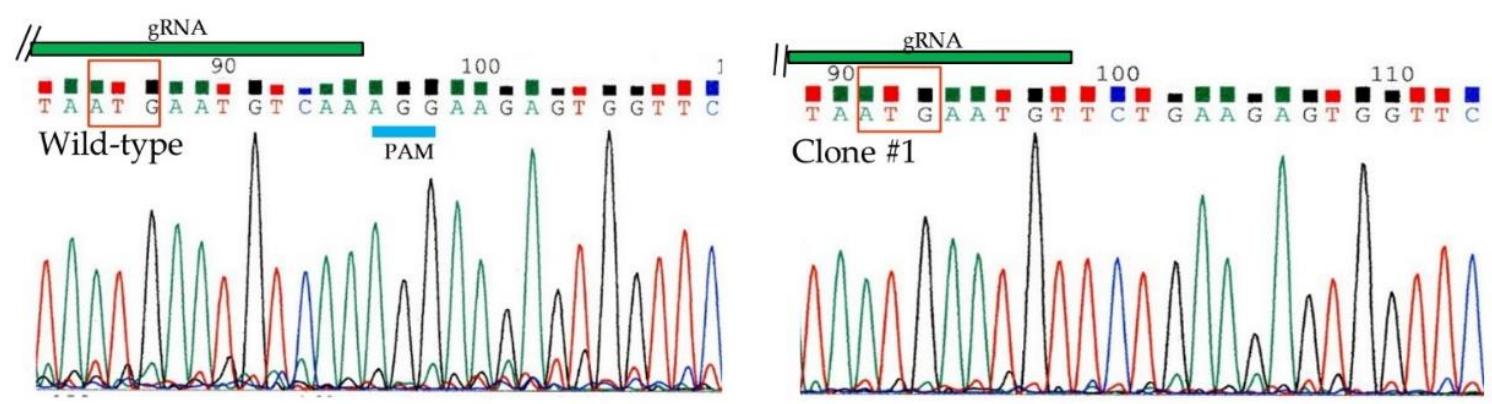

Figure 3 Characterization of the EGFP-expressing genome-edited porcine clones. A. Clones \#1 (a, b) and \#8 (c, d), and the cells transfected with pTrans and pT-EGFP (emf). Although both clones exhibited loss of $\alpha$-Gal epitope expression (bad; determined by staining with AF594-IB4), Clone \#1 exhibited a uniform expression of EGFP (a), while Clone \#8 presented a mixture of EGFP-positive (arrows in c) and EGFP-negative cells (arrowheads in $\mathrm{c}$ ), thereby demonstrating a mosaic EGFP expression. In contrast, the cells transfected with pTrans and pT-EGFP exhibited (four days later) mosaic EGFP expression and ubiquitous $\alpha$-Gal epitope expression on their cell surface. Fluorescence was examined under UV and visible light. Scale bar $=25 \mu \mathrm{m}$. B. Ideograms of the EGFPexpressing genome-edited porcine cells (Clone \#1) and wild-type cells (Wild-type) obtained after direct sequencing of the nested PCR products using the Ex4-2S primer. ATG is depicted inside a red box. The PAM site is represented with a blue line. 
Table 1 Characterization of the gene-modified clones obtained using pipette tip-aided cell cloning.

\begin{tabular}{|c|c|c|c|c|}
\hline $\begin{array}{l}\text { Name of } \\
\text { clones }^{1}\end{array}$ & $\begin{array}{l}\text { Pattern of mutations } \\
\text { in GGTA1 exon } 4^{2}\end{array}$ & Sequence of porcine GGTA1 exon $4^{3}$ & Expression of EGFP 4 & $\begin{array}{l}\alpha \text {-Gal epitope } \\
\text { expression }^{5}\end{array}$ \\
\hline wt & 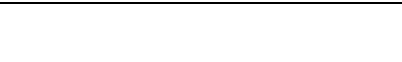 & AATAATGAATGT-- & 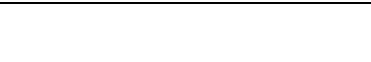 & \\
\hline \multirow{3}{*}{$\# 1$} & \multirow{3}{*}{ Bi; Replac } & CAAAGGAAGAGTGGTTCTGTCAAT & - & + \\
\hline & & 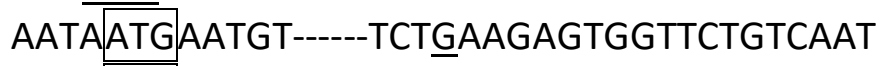 & \multirow{2}{*}{+} & \multirow{2}{*}{-} \\
\hline & & AATA ATGAAT----CAAAGGAAGAGTGGTTCTGTCAAT & & \\
\hline \#2 & $\mathrm{Bi} ;$ Del & AATAATGAATGTTTCAAAGGAAGAGTGGTTCTGTCA & + & - \\
\hline \#3 & $\mathrm{Bi}$; Ins & AT & + & - \\
\hline \#4 & $\mathrm{Bi}$; Ins & AATAATGAATGTT- & + & - \\
\hline \#5 & - & CAAAGGAAGAGTGGTTCTGTCAAT & + & - \\
\hline \#6 & - & ND & + & - \\
\hline \#7 & - & ND & + & - \\
\hline \#8 & - & ND & Mosaic & - \\
\hline \#9 & - & ND & - & - \\
\hline$\# 10$ & - & ND & + & - \\
\hline \#11 & - & ND & + & - \\
\hline \#12 & - & ND & Mosaic & - \\
\hline$\# 13$ & - & ND & - & - \\
\hline \#14 & - & ND & + & - \\
\hline \#15 & - & ND & + & - \\
\hline \multirow[t]{2}{*}{$\# 16$} & - & ND & + & - \\
\hline & & ND & & \\
\hline
\end{tabular}

${ }^{1}$ In vitro electroporation of porcine fibroblasts was performed in the presence of RNPs (targeted to porcine GGTA1) and piggyBac transposons. The treated cells were subjected to toxin-based selection to obtain viable cell colonies lacking $\alpha$-Gal epitope expression. The obtained single colony-derived cells were then examined for EGFP-derived fluorescence. A few clones were also subjected to molecular 
biology analysis to identify the possible mutations in the GGTA1 target region. Staining with AF594-IB4 revealed that all of the clones tested were negative for $\alpha$-Gal epitope expression.

${ }^{2}$ Mutations in exon 4 of GGTA1 are defined as Ins (insertion of nucleotide [s]), Del (deletion of nucleotide [s]), and Replac (deletion of nucleotide [s] and subsequent insertion of nucleotide [s]). Bi, bi-allelic KO mutations.

${ }^{3}$ Clonal sequences of exon 4 of GGTA1, including ATG site (boxed), are depicted. The PAM site is underlined.

${ }^{4}$ EGFP-derived fluorescence was examined using a fluorescence microscope. The cells exhibiting uniform EGFP expression are indicated with "+," while the cells exhibiting no EGFP expression are indicated with "-." The colonies comprising a mixture of fluorescent and nonfluorescent cells were defined as a mosaic.

${ }^{5}$ Based on the staining of clones with AF594-IB4, the cells stained positively are indicated with "+", while the cells exhibiting no $\alpha$-Gal epitope expression are indicated with "-".

wt, wild-type cells; ND, not determined. 
Furthermore, the possible occurrence of mutations (indels) involving the GGTA1 locus was also assessed through the direct sequencing of the PCR products ( $350 \mathrm{bp}$ ) spanning the sequence recognized by gRNA. As depicted in the left panel of Figure $3 B$, the wild-type unedited porcine cells had the authentic GGTA1 DNA sequence. In contrast, Clone \#1, a clone identified as EGFP-positive and $\alpha$-Gal epitope-negative, exhibited an alteration in the nucleotides (from CAAAG to TCT), including those at the PAM site (right panel in Figure 3B). Owing to the clear pattern observed in the ideograms, Clone \#1 appears to represent the cells with a homozygous KO phenotype. In regard to the occurrence of possible mutations in the other clones, the tested clones exhibited biallelic phenotypes for the mutations involving GGTA1 (Table 1), which was in agreement with the results of cytochemical staining with AF594-IB4.

Several cell cloning methods, including cell dilution method [28], steel ring-aided method [29], fluorescence-activated cell sorting (FACS) [30], and paper-aided method, have been developed [3], and all these approaches have their own advantages and disadvantages. For instance, the cell dilution method, which has been used widely as an effective cell cloning method and requires cell counting and calculation to allow for the seeding of a single cell into each well of a 96-well plate, may prove to be inadequate when the cells fail in the clonal expansion which they often do. The steel ring-aided method is another traditional technique that utilizes sterile steel rings for the isolation of trypsinized cells as well as sterile grease to facilitate tight attachment between the ring and the substratum of the dish, and it is often difficult to collect several cell clones (>30) at once. FACS is effective for the collection of target cell clones, although it often damages the isolated cells, and the apparatus required is expensive. In this context, the paper method appears to be an attractive alternative due to its simplicity compared to the aforementioned three methods and the possibility of collecting $>30$ clones at once [3]. However, even the paper method (using Whatman ${ }^{\circledR}$ 3MM paper) was unsuitable for certain types of porcine cells, for which $>70 \%$ of clones fail to survive after aspirating the cells. However, the pipette tip-aided method proposed in the present study was demonstrated to be useful in the isolation of these cloning-sensitive porcine cells, as $>80 \%$ of the isolated cells in the present study successfully survived after cloning. In addition, it was possible to aspirate the cells from $>30$ colonies within $10 \mathrm{~min}$. However, beyond $10 \mathrm{~min}$, the cells could be damaged, probably due to water loss from the surface of the culture dish. Therefore, to acquire more colonies, a similar approach using another dish could be used. Notably, a similar approach was reported by $\mathrm{Na}$ et al. [31], who attempted to isolate colonies from $\mathrm{GM}$ embryonic stem cells (ESCS). The authors grew the colonies on feeder cells, treated them with trypsin for 0.5$1 \mathrm{~min}$ to detach the colonies from the plate, and then covered the treated cells with medium to inactivate trypsin. These colonies were then picked using a glass capillary fitted to a mouthpiece under a dissecting microscope. It was observed that approximately $16 \%$ of the picked clones were mosaic, probably due to the presence of at least two clones during colony formation.

Our ultimate objective of the present study was to demonstrate that the proposed pipette tipaided method is useful in the isolation of GM porcine clones generated upon transfection and subsequent toxin-based selection. In order to verify the validity of this approach, porcine fibroblasts were co-transfected with two components - one for the destruction of GGTA1 via CRISPR/Cas9-based genome-editing and the other for enabling chromosomal integration of a GOI via the PB gene-delivery system. The transfected cells were subjected to toxin-based selection using IB4SAP. The emerging colonies were picked using the pipette tip-aided method. All 16 isolated clones survived and proliferated well. Therefore, the survival rate of the clones isolated 
using the proposed method appears to be 100\%. No AF594-IB4-positive clones were observed among the 16 isolated clones (Figure 3A-b, d; Table 1), suggesting bi-allelic KO of the GGTA1 locus, which was also confirmed by direct sequencing of the selected clones [Clone \#1 in Figure 3B (right)]. This indicated that the IB4SAP-based selection system was effective. Furthermore, almost all clones (75\%) expressed EGFP uniformly (Figure 2B-b and $3 A-a$; Table 1), while the remaining ones exhibited mosaic or negative EGFP expression (Figure 3A-C; Table 1). The appearance of EGFP-negative clones could be attributed to the failure of chromosomal integration of $P B$ transposons into the host cell genome upon co-transfection. The presence of clones exhibiting mosaic EGFP expression could be attributed to contamination with EGFP-negative cells during the cloning process.

One could wonder if the proposed tip-aided approach for isolating single colonies would be effective for other cell lines or the cells with genome-edited loci other than GGTA1. This query could be resolved by referring to a few previous reports. Watanabe et al. [27] employed a paper method [3] for isolating colonies and demonstrated the successful destruction of the DiGeorge syndrome critical region gene 2 ( Dgcr2) locus in mouse ESCs after co-transfection with the vectors carrying the Cas9 gene and the gRNA (targeted to Dgcr2), introduction of the gene encoding endo$\beta$-galactosidase (capable of cleaving the $\alpha$-Gal epitope), and subsequent selection using IB4SAP. Furthermore, the transforming growth factor- $\beta$ receptor type 1 (TGFBRI) gene was successfully destroyed in porcine adipocyte precursor cells (PAPCS) using similar technology. These findings suggest the effectiveness of the tip-aided approach for colony isolation and that genome editing is possible at other loci and in other cell types (i.e., mouse ESCs and PAPCs) as well.

In conclusion, a novel pipette tip-aided cell collection technique was developed in the present study for acquiring viable GM cell clones with a $100 \%$ survival rate. The proposed method is simple and convenient, rendering it suitable for wide application in the large-scale isolation of GM mammalian clones (including humans) if the cells survive and grow as viable colonies after the transfection and subsequent selection.

\section{Acknowledgments}

This study was partly supported by a grant (no. $19 \mathrm{~K} 06372$ for M.S.; no. 18 K09839 for E.I.) from The Ministry of Education, Science, Sports, and Culture, Japan.

\section{Author Contributions}

M.S. conceived and designed the study, drafted the manuscript, performed the experiments, and revised the manuscript. I.S., E.A., and E.I. critically revised the manuscript.

\section{Funding}

This article received no external funding.

\section{Competing Interests}

The authors have declared that no competing interests exist. 


\section{References}

1. Martin BM. Tissue culture techniques: An introduction. Cambridge, MA: Birkhäuser Boston; 1994. pp. 143-151.

2. McFarland DC. Preparation of pure cell cultures by cloning. Methods Cell Sci. 2000; 22: 63-66.

3. Nakayama A, Sato M, Shinohara M, Matsubara S, Yokomine T, Akasaka E, et al. Efficient transfection of primarily cultured porcine embryonic fibroblasts using the Amaxa nucleofection system ${ }^{\mathrm{TM}}$. Cloning Stem Cells. 2007; 9: 523-534.

4. Horvath P, Barrangou R. CRISPR/Cas, the immune system of bacteria and archaea. Science. 2010; 327: 167-170.

5. Bhaya D, Davison M, Barrangou R. CRISPR-Cas systems in bacteria and archaea: Versatile small RNAs for adaptive defense and regulation. Annu Rev Genet. 2011; 45: 273-297.

6. Jinek M, Chylinski K, Fonfara I, Hauer M, Doudna JA, Charpentier E. A programmable dualRNA-guided DNA endonuclease in adaptive bacterial immunity. Science. 2012; 337: 816-821.

7. Gaj T, Gersbach CA, Barbas III CF. ZFN, TALEN, and CRISPR/Cas-based methods for genome engineering. Trends Biotechnol. 2013; 31: 397-405.

8. Harrison MM, Jenkins BV, O'Connor-Giles KM, Wildonger J. A CRISPR view of development. Genes Dev. 2014; 28: 1859-1872.

9. Hsu PD, Lander ES, Zhang F. Development and applications of CRISPR-Cas9 for genome engineering. Cell. 2014; 157: 1262-1278.

10. Sykes M, Sachs DH. Transplanting organs from pigs to humans. Sci Immunol. 2019; 4: eaau6289.

11. Sandrin MS, McKenzie IF. Gal alpha $(1,3)$ Gal, the major xenoantigen(s) recognised in pigs by human natural antibodies. Immunol Rev. 1994; 141: 169-190.

12. Akasaka E, Watanabe S, Himaki T, Ohtsuka M, Yoshida M, Miyoshi K, et al. Enrichment of xenograft-competent genetically modified pig cells using a targeted toxin, isolectin BS-I-B 4 conjugate. Xenotransplantation. 2010; 17: 81-89.

13. Sato M, Akasaka E, Saitoh I, Ohtsuka M, Nakamura S, Sakurai T, et al. Targeted toxin-based selectable drug-free enrichment of mammalian cells with high transgene expression. Biology. 2013; 2: 341-355.

14. Ivics Z, Li MA, Mátés L, Boeke JD, Nagy A, Bradley A, et al. Transposon-mediated genome manipulation in vertebrates. Nat Methods. 2009; 6: 415-422.

15. Ivics Z. Endogenous transposase source in human cells mobilizes piggyBac transposons. Mol Ther. 2016; 24: 851-854.

16. Wilson MH, Coates CJ, George Jr AL. PiggyBac transposon-mediated gene transfer in human cells. Mol Ther. 2007; 15: 139-145.

17. Bai DP, Yang MM, Chen YL. PiggyBac transposon-mediated gene transfer in Cashmere goat fetal fibroblast cells. Biosci Biotechnol Biochem. 2012; 76: 933-937.

18. Li R, Zhuang Y, Han M, Xu T, Wu X. PiggyBac as a high-capacity transgenesis and gene-therapy vector in human cells and mice. Dis Model Mech. 2013; 6: 828-833.

19. Inada E, Saitoh I, Watanabe S, Aoki R, Miura H, Ohtsuka M, et al. PiggyBac transposonmediated gene delivery efficiently generates stable transfectants derived from cultured primary human deciduous tooth dental pulp cells (HDDPCs) and HDDPC-derived iPS cells. Int J Oral Sci. 2015; 7: 144-154. 
20. Sato M, Maeda K, Koriyama M, Inada E, Saitoh I, Miura H, et al. The piggyBac-based gene delivery system can confer successful production of cloned porcine blastocysts with multigene constructs. Int J Mol Sci. 2016; 17: 1424.

21. Vaughan HA, Loveland BE, Sandrin MS. Gal alpha $(1,3)$ Gal is the major xenoepitope expressed on pig endothelial cells recognized by naturally occurring cytotoxic human antibodies. Transplantation. 1994; 58: 879-882.

22. Sato $M$, Miyoshi K, Nagao $Y$, Nishi $Y$, Ohtsuka M, Nakamura S, et al. The combinational use of CRISPR/Cas9-based gene editing and targeted toxin technology enables efficient biallelic knockout of the $\alpha-1$, 3-galactosyltransferase gene in porcine embryonic fibroblasts. Xenotransplantation. 2014; 21: 291-300.

23. Sato $M$, Inada E, Saitoh I, Matsumoto $Y$, Ohtsuka $M$, Miura $H$, et al. A combination of targeted toxin technology and the piggyBac-mediated gene transfer system enables efficient isolation of stable transfectants in nonhuman mammalian cells. Biotechnol J. 2015; 10: 143-153.

24. Ohtsuka $M$, Sato $M$, Miura $H$, Takabayashi $S$, Matsuyama $M$, Koyano $T$, et al. i-GONAD: A robust method for in situ germline genome engineering using CRISPR nucleases. Genome Biol. 2018; 19: 25.

25. Hitoshi N, Ken-ichi $\mathrm{Y}$, Jun-ichi M. Efficient selection for high-expression transfectants with a novel eukaryotic vector. Gene. 1991; 108: 193-199.

26. Sato M, Akasaka E, Saitoh I, Ohtsuka M, Nakamura S, Sakurai T, et al. A simplified protocol for the semi-large scale recovery of plasmids from Escherichia coli grown on agar plates. J Biomed Sci Eng. 2012; 5: 406-408.

27. Watanabe S, Sakurai T, Nakamura S, Miyoshi K, Sato M. The combinational use of CRISPR/Cas9 and targeted toxin technology enables efficient isolation of bi-allelic knockout non-human mammalian clones. Int J Mol Sci. 2018; 19: 1075.

28. Puck TT, Marcus PI. A rapid method for viable cell titration and clone production with HeLa cells in tissue culture: The use of X-irradiated cells to supply conditioning factors. Proc Natl Acad Sci U S A. 1955; 41: 432-437.

29. Domann R, Martinez J. Alternative to cloning cylinders for isolation of adherent cell clones. Biotechniques. 1995; 18: 594-595.

30. Julius MH, Masuda T, Herzenberg LA. Demonstration that antigen-binding cells are precursors of antibody-producing cells after purification with a fluorescence-activated cell sorter. Proc Natl Acad Sci U S A. 1972; 69: 1934-1938.

31. Na E, Landsberger A, Manz S, Urban I, Wittler L, Michel G. A new method to pick targeted ES cell clones more efficiently. Transgenic Res. 2014; 23: 846. 
OBM Genetics 2021; 5(1), doi:10.21926/obm.genet.2101126

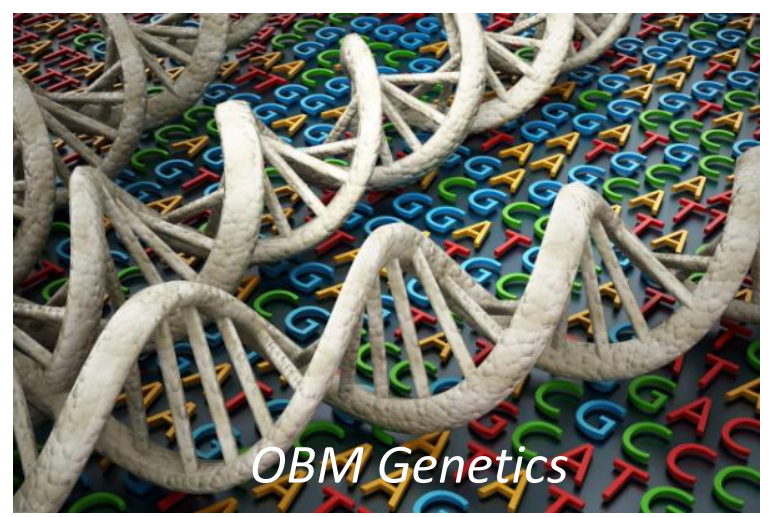

Enjoy OBM Genetics by:

1. Submitting a manuscript

2. Joining in volunteer reviewer bank

3. Joining Editorial Board

4. Guest editing a special issue

For more details, please visit:

http://www.lidsen.com/journals/genetics 